\title{
Dinámica de la población gaditana en el siglo XVIII: una reconstrucción crítica a partir de las fuentes
}

\author{
JUIO PEREZ SERRANO
}

\section{INTRODUCCION}

La dinámica de la población gaditana a lo largo de todo el siglo XVIII no ha sido hasta ahora estudiada monográficamente, si bien contamos con diversas aproximaciones parciales y contribuciones en el marco de trabajos de carácter más general (1). Por otro lado, el mayor interés despertado por el último cuarto del siglo, ya desde las primeras décadas de la centuria decimonónica, ha concentrado las investigaciones de este último período (2), buscando a menudo contribuir a desvelar las causas y dimensionar los efectos de la crisis comercial finidieciochesca, lo que ha repercutido negativamente sobre el desarrollo de nuestro conocimiento de la etapa anterior. $\mathrm{El}$ número y la profundidad de las publicaciones específicamente demográficas referidas a las ocho décadas que van de 1693 a 1773 son tan limitados, que cabría hablar de un auténtico "páramo" historiográfico (3). Por contra, repetidamente los testimonios coetáneos y la historiografía

(1) Por ejemplo, entre las primeras, Vid. PONCE CORDONES, F.: "Dos siglos claves en la demografía gaditana (Breve estudio sobre la evolución de la población de Cádiz en las centurias XVII y XVIII". Gades, 11 (1983), pp. 417-453. Entre las segundas, Vid. PASCUA SANCHEZ, M². J. de la: "Cádiz y su provincia, 1700-1788". En: VARIOS: Cádiz y su provincia, II. Sevilla, 1984, pp. 293 ss.

(2) Vid. PEREZ SERRANO, J.: La población de Cádiz a fines del Antiguo Régimen. Su estructura y mecanismos de renovación (1775-1800). Cádiz, 1989.

(3) Un desierto que ya empieza a desbrozarse con la investigación en curso de J.M. Molina Martínez, referida al período 1700-1775, cuyas conclusiones habrán sin duda de contribuir a llenar eficazmente el actual vacío. 
posterior de carácter más general, las descripciones literarias y las propias fuentes demográficas, coinciden en poner de manifiesto la gran importancia del aspecto poblacional en el esplendor gaditano a lo largo de todo el XVIII.

En efecto, los movimientos de la población gaditana en los tres últimos siglos se han ajustado con bastante fidelidad a las fluctuaciones de su economía mercantil - "y es que pocas son las ciudades que han estado tan estrechamente vinculadas al tráfico marítimo como Cádiz" (4)-, por lo que el análisis demográfico se convierte de esta forma en una clave para la correcta interpretación de la historia de Cádiz en su conjunto. Teniendo presente esta doble dimensión, abordamos aquí el diseño de las principales líneas del perfil demográfico gaditano a lo largo del siglo XVIII, años en los que Cádiz continúa el crecimiento iniciado ya, de manera atípica por lo que se refiere a otras regiones y, en general, al ámbito español, en las primeras décadas de la anterior centuria (5).

Restaurada la armonía con la dinámica global de la población española, que desde comienzos del XVIII había logrado afianzar también una tendencia al crecimiento ininterrumpido, la población gaditana manifestará sin embargo una cierta recesión en torno al cambio de siglo, debido a la estrecha conjunción de los factores malthusianos que caracterizaron en España la coyuntura crítica del Setecientos (6). A diferencia de otras regiones españolas - la propia Andalucía- donde la dinámica natural es responsable del crecimiento demográfico, Cádiz basará su desarrollo a lo largo de los siglos XVII y XVIII en el aporte masivo de recursos humanos procedentes no sólo de su entorno inmediato, sino de los más variados lugares de Europa y del mundo. De ahí que el cambio de tendencia en la coyuntura económica perceptible a finales del XVIII, vinculado a la crisis del comercio americano, conllevara la fuerte caída en principio y el posterior estancamiento del número de habitantes: el sentido y, sobre todo, las dimensiones del flujo migratorio habían sufrido una importante mutación.

(4) BERNAL, A.M. -COLLANTES DE TERAN, A.-GARCIA-BAQUERO, A.: "Sevilla: de los gremios a la industrialización". Estudios de Historia Social, 5-6 (1978), p. 33.

(5) La población gaditana se triplica en la primera mitad'del siglo XVI, pasando de 7.100 a 21.900 habitantes, Vid. PORQUICHO MOYA, I.: Cádiz: Población y Sociedad, 1597-1650. Los libros parroquiales. Sevilla, 1982, tesis de licenciatura inédita.

(6) La Guerra de Sucesión, las frecuentes epidemias y las hambrunas que se extienden por el país en la primera década del XVIII. 
Trataremos de reconstruir aquí las tres grandes fases en que, a nuestro juicio, es posible parcelar la evolución demográfica gaditana durante el XVIII: a) la inestabilidad que caracteriza las primeras décadas del siglo; b) el crecimiento sostenido en la plenitud de la centuria; y c) la ralentización y definitiva crisis que tiene lugar en el último cuarto del siglo. Englobaremos, por tanto, la primera fase de la etapa proto-estadística, que se inicia en Cádiz en 1700 con las reformas administrativas introducidas por los Borbones y se extiende aproximadamente hasta 1835 (7). No obstante, el estadio todavía muy incipiente de la estadística hasta 1787 justifica, como veremos, que las cifras aportadas por los censos y padrones anteriores a esa fecha (8) deban ser tomadas con mucha prevención, cuando no abiertamente rechazadas.

\section{LOS PROBLEMAS DEL CAMBIO DE SIGLO}

Los recuentos de población y otras fuentes conservadas reflejan un claro incremento de la población a lo largo del siglo XVIII, continuando la tendencia manifestada ya durante toda la centuria anterior (9). Sin embargo, entre ambas fases se constata un breve paréntesis depresivo, coincidiendo precisamente con los inicios del nuevo siglo, época turbulenta marcada por la crisis política y económica originada en España tras la muerte sin sucesión del último Austria, Carlos II. La documentación no deja resquicio a la duda sobre la existencia de este retroceso, que parece demostrado, pero los problemas comienzan cuando intentamos evaluar en su justa medida las dimensiones reales de la crisis demográfica, dada la finalidad puramente recaudatoria de los recuentos que se conservan.

(7) Desde entonces se regularizará la confección de padrones anuales (a partir de 1837) y se instaurará en Cádiz el Registro Civil (1840-41), inaugurando así la etapa estadística.

(8) Sobre los padrones anteriores a 1786, Vid.. VARIOS: "Fuentes para la demografia de Cádiz (siglos XVIII-XIX)". Gades, 11. (1983), pp. 385-387. Más voluminoso, pero sin aportar prácticamente nada nuevo, Vid. ESPINOSA DE GODOS, E.: Cádiz, lonja europea en el siglo XVIII (Población y Sociedad). Sevilla, 1984, tesis doctoral inédita.

(9) La tasa media de crecimiento anual fue durante el XVI extraordinariamente alta, 3'1\%, lo que permitió a Cádiz multiplicar quince veces sus efectivos desde comienzos del siglo, Cfr. BERNAL, A.M. -COLLANTES DE TERAN, A.- GARCIA-BAQUERO, A.: "Sevilla..." op. cit., p. 27. 


\subsection{Lo que constatan las fuentes}

Entre 1709 y 1714 se elaboran cuatro estadísticas sucesivas y con el fin de evaluar las posibilidades de obtener fondos para apoyar la causa de Felipe $V$ en la guerra de Sucesión. Todas incluyen en sus estados, como es habitual en los padrones fiscales, sólo a los vecinos cabezas de familia y a los extranjeros avecindados, indicando la nacionalidad de origen; se añaden también, excepto en 1714, los eclesiásticos, aunque sin contabilizar, por no estar sometidos a la contribución. El padrón de 1709, que forma parte del empadronamiento general conocido por "calle-hita", da un total de 4.932 vecinos, excluidos los eclesiásticos (10). Su vigencia será muy corta, pues inmediatamente después de la elaboración del padrón caerán sobre la ciudad dos grandes calamidades que provocarán un fuerte deterioro en su vecindario: la grave epidemia de 1709 y la tremenda crisis de subsistencias iniciada ese mismo año. Muerte y emigración harán disminuir sensiblemente los efectivos humanos(11); de ahí que se plantee la necesidad de actualizar la estadística para proceder al cobro de la contribución de guerra.

Resultado de ello fue el padrón de 1710, que arroja un total de 4.043 vecinos, también con exclusión, como decimos, de los eclesiásticos. Tres años después, de nuevo por orden real y con el mismo fin que los anteriores, se confeccionará un padrón de vecinos naturales y extranjeros, cuyo estado general no se diferencia mucho del anterior de 1710: en 1713 se contabilizan 4.048 vecinos (12). Pero, todavía poco antes de finalizar la guerra, en junio de 1714, Felipe $V$ mandará realizar un nuevo recuento del vecindario para distribuir un último y definitivo impuesto de guerra, cuyo cobro será finalmente suspendido en 1715 , terminado el conflicto (13). La relación que aporta, sin duda muy incompleta, incluye 1.573 naturales y 520 extranjeros, en total 2.093 vecinos (14), a todas luces un dato irreal. Con él concluimos la presentación de los cómputos directos con que contamos para reconstruir la dinámica demográfica gaditana en las dos primeras décadas de la centuria.

(10) Cfr. A.M.C. Sección Padrones. Leg. 1002. "Padrón de 1709".

(11) En la presentación del padrón se dirá que "[...] con motivo de las azeleradas y repentinas enfermedades que vinieron, fallecieron en esta ciudad muchos vecinos y otros [...] para poderse mantener a caussa de la gran carestía del pan se aussentaron de ella con su familia [...]", A.M.C. Sección Padrones. Leg. 1.002. "Padrón de 1710".

(12) Cfr. A.M.C. Sección Padrones. Leg. 1.003. "Padrón de 1713".

(13) Cfr. VARIOS: "Fuentes para la demografia de Cádiz..." op. cit., p. 386.

(14) Cfr. A.M.C. Sección Padrones. Leg. 1.004. "Padrón de 1714". 
Observando el último recuento llevado a cabo en el siglo XVII, el de 1693, confeccionado para la leva militar, que nos da la cifra de 5.191 vecinos (15), podemos percibir con claridad, a tenor de las cifras aportadas por los padrones de comienzos del XVIII —sometidos a idénticas determinaciones-, la existencia de una contracción demográfica en las primeras décadas del nuevo siglo. Ni que decir tiene que, por su finalidad, en éste, como en los anteriormente citados, han de concurrir de manera obligada numerosas omisiones (los "exentos") y ocultaciones. Por ello; aunque la recesión es incuestionable, el valor absoluto de las cifras demanda una reflexión más detenida.

\subsection{Una interpretación de los datos}

Al comparar las cifras que se obtienen estableciendo tasas variables (16) para las series biológicas de $1693,1709,1710,1713$ y 1714, y las resultantes de aplicar a los datos aportados por los padrones vecinales de esos años el coeficiente 5 para la reconversión de vecinos en habitantes (máximo aceptable, a nuestro juicio, para esta etapa) (17), comprobamos que entre ambas series existen dos diferencias fundamentales: el volumen global de las cifras, muy superior en la calculada por aplicación de tasas variables a nacimientos y matrimonios, y la tendencia demográfica que manifiestan. Veamos a continuación el resultado de este contraste en una tabla:

TABLA 1

POBLACION DE CADIZ ENTRE 1693 Y 1714

\section{Aplicando el coeficiente}

a los padrones vecinales

$$
\begin{aligned}
& 29.955 \\
& 24.660 \\
& 20.215 \\
& 20.240
\end{aligned}
$$$$
10.465(1)
$$

Años

1693

1709

1710

1713

1714
Aplicando tasas variables a las series parroquiales

42.500

30.000

32.500

36.500

39.500

(15) Cfr. A.G.S. Sección Guerra Antigua. Leg. 2.934. "Padrón de Cádiz de 1693".

(16) Esta estrategia fue ya propuesta por F. Ponce en "Dos siglos claves..." op. cit., p. 433. No coincidimos, sin embargo, en las tasas aplicadas; nosotros usamos $\mathrm{N}_{\mathrm{r}}=31$ '5 por mil para el siglo XVII, para el XVIII $\mathrm{N}_{\mathrm{r}}=28{ }^{\prime} 5$ por mil y $\mathrm{C}_{\boldsymbol{r}}$ móvil oscllando entre $6 ' 5$ y $11 ' 5$ por mil.

(17) Vid. BUSTELO GARCIA DEL REAL, F.: "La transformación de vecinos en habitantes. El problema del coeficiente". Estudios Geográficos, XXXIII/130 (1973) PP. 154-164. Ni siquiera empleando el coeficiente 6 , adeucado sólo para los deficitarios censos del XVI, lograríamos igualar las cifras obtenidas por el sistema de tasas variables. 
Respecto al volumen global de la población, ya lo hemos indicado, los padrones carecen de utilidad para conocer los valores absolutos; las diferencias entre ambas series oscilan entre el 22 y el 80 por ciento, dejando a un lado, por supuesto, el dato del padrón vecinal de 1714, rechazable de antemano. Mucho mayores serían aún las distancias si, como hace $\mathrm{M}^{2} \mathrm{~J}$. de la Pascua, empleásemos en la reconversión el coeficiente 4 (18).

Por lo que se refiere a la tendencia, habría que realizar algunas precisiones. En primer lugar, constatar que el padrón de 1709 se realiza antes de que tuviera lugar la grave epidemia que asoló la ciudad en ese año (19), por lo que no supone una gran caída respecto a 1693; quizás el 5 por ciento a que ascienden las pérdidas pueda explicarse simplemente por la propia complejidad del periodo, sin recurrir a la hipotética epidemia de fiebre amarilla de 1705 , cuya existencia no hemos logrado comprobar (20). Por el contrario, el cálculo realizado sobre las series parroquiales refleja los efectos de la epidemia en el mismo año de 1709; de ahí que la rebaja sea cercana al 30 por ciento. Queda así resuelta la primera contradicción.

El mismo razonamiento ayuda a interpretar un segundo contraste, los valores divergentes de 1710 , pues mientras el padrón recoge ahora, en un contexto depresivo de la economía, la regresión demográfica experimentada el año anterior, las series biológicas del año reflejan ya la actitud de cierto optimismo por el final de las calamidades que debió caracterizar los últimos meses del año, por otra parte, los de mayor natalidad. Así se explica lo que en apariencia es otra contradicción: que el padrón de 1710 manifieste un retroceso poblacional del 18 por ciento respecto a 1709 , cuando aplicando las tasas a las series se refleja una cierta recuperación, estimable en torno al 8 por ciento.

Así mismo, lamentamos no poder compartir la extrañeza de los profesores Bernal, Collantes y García-Baquero ante la cifra aportada por el padrón de 1713, cuando acusan "la desaparición, en sólo cuatro

(18) Vid. PASCUA SANCHEZ, $M^{*} J$. de la: "Cádiz y su provincia, 1700-1788". En: VARIOS: Cádiz y su provincia, II. Sevilla, 1984, p. 291.

(19) Probablemente se tratase del vómito negro, aunque existen dudas sobre su etiologia, Cfr. PONCE CORDONES, F.: “Dos siglos claves...” op. cit., p. 429.

(20) J. Nadal difunde la información, que procede de una fuente historiográfica, Vid. GONZALEZ DE SAMANO, M.: Monografía bistórica del cólera-morbo asiático, 1. Madrid, 1858, p. 31. 
años, de un total de 884 vecinos, lo que significa una pérdida del 18 por 100 con respecto al padrón inmediatamente anterior (sic) y del 22 por 100 respecto al de 1693, en unos momentos, además, que no tenían por qué revestir unas características especialmente negativas para Cádiz" (21). Esta cita demanda alguna precisión, pues no es cierto que entre 1709 y 1713 no medie estadística alguna: se conserva el padrón fechado en 1710. Pero la trascendencia de esta omisión emana de que es justo en este recuento donde se halla la clave para entender el precario estado de 1713 , pues en él se plasman con claridad los efectos de un factor que sí es "especialmente negativo" para la población gaditana: la epidemia de 1709 , fundamental para reconstruir la demografía de este período (22).

Por último, la similitud entre las cifras de los padrones de 1710 y 1713 no se corresponde con lo expresado por las series, donde se observa una sensible recuperación en el número de habitantes, sin duda por efecto del cambio de coyuntura. No obstante, aunque en estos años el crecimiento demográfico no permaneció paralizado, coincidimos plenamente con Ponce en rechazar la generalizada idea de que "la regresión de los años 1710 a 1712 fue sólo aparente y originada porque los padrones [...] comportaban notables ocultaciones y falseamientos de datos" (23). Con seguridad hubo falseamiento y ocultaciones, pero, tan evidente como esto es que, por la conjunción de factores mencionados, durante las dos primeras décadas del siglo XVIII vivió Cádiz una fase de estancamiento demográfico, que sólo pudo remontar con firmeza a partir de 1720 . Desde ese momento, con ligeras ralentizaciones, se mantendrá el crecimiento sostenido hasta la última década de la centuria.

\section{LA PLENITUd DEL SIGLO}

\subsection{Un repaso a las fuentes}

Desaparecidos los frenos que impedían el crecimiento en años anteriores, la población gaditana reinicia la expansión. Hasta mediados de siglo no contamos con una estadística general fiable, el Catastro de

(21) BERNAL, A.M. -COLLANTES DE TERAN, A.- GARCIA-BAQUERO, A.: "Sevilla...", op. cit., p. 31.

(22) Cfr. OROZCO ACUAVIVA, A.: Apuntes para la Historia de la Medicina gaditana, Cádiz, 1970, p. 52.

(23) PONCE CORDONES, F.: "Dos siglos claves..." op. cit., p. 129. 
Ensenada (1752), y hasta 1769 no aparece el primer censo realizado contando individuos en lugar de vecinos, el ordenado por el conde de Aranda. A estas informaciones habria que añadir los datos, muy criticados (24), del Vecindario de Campoflorido (1717) y de la Theórica y práctica de Comercio y de Marina (1724), de J. de Ustáriz, así como los resultados del padrón vecinal de 1773 , ejecutado para proveer la quinta. Veamos qué nos aportan estas estadísticas.

En 1717 se contabilizan 4.043 vecinos (25), los mismos que en 1710 , lo que no resulta muy asumible siquiera sea en términos relativos, pues, aunque sabemos que Cádiz se recuperó lentamente de la crisis, es difícil aceptar que siete años después la población se mantuviese todavía en la cota mínima a que se había visto reducida en el momento álgido de la depresión. La cifra de 7.100 vecinos que Ustáriz atribuye a $1723(26)$, pese a no proceder del recuento directo, sino de una modificación de las cifras del Vecindario de Campoflorido, resulta mucho más verosímil, pues eleva sensiblemente la magnitud de los efectivos demográficos gaditanos.

A mediados de siglo, en 1752 (27), se constatan ya documentalmente 9.842 vecinos, lo que confirma la tendencia ascendente que había tratado de poner de manifiesto Ustáriz en su Theórica. La primera estimación sobre el total de "almas", correspondiente a 1769 (28), vuelve a demostrar, ahora con rotundidad, el fuerte ritmo del crecimiento demográfico, pues asigna a Cádiz 64.838 individuos; 12.968 vecinos si, como hemos venido haciendo, aplicamos el coeficiente 5 para la reconversión.

(24) Vid. BUSTELO GARCIA DEL REAL, F.: "Algunas reflexiones sobre la población española de principios del siglo XVIII". Anales de Economía, 15 (1972), pp. 89-106; IDEM: "El Vecindario General de España de 1712-17 ${ }^{\circ} O$ Censo de Campoflorido [II]". R.I.S., XXXIII (1974), pp. 16-35; MARTIN GALAN, M.: "Fuentes y métodos para el estudio de la demografía histórica castellana durante la Edad Moderna". Hispania, 148 (1981), p. 252.

(25) Cfr. B.N., Sección Manuscritos, ms. 2.274. "Vecindario General de Campoflorido y Ustáriz, 1712-1717".

(26) Cfr. USTARIZ, J. de: Theórica y Práctica de Comercio y de Marina, Madrid, 1742², cap. XVII, pp. 34-39. Ustáriz incrementa alrededor de un 75 por ciento los datos de 1717 relativos a Cádiz, y utiliza el coeficiente 5 para transformar vecinos en habitantes.

(27) Cfr. A.G.S., Dirección General de Rentas, primera remesa. Leg. 2.046. "Vecindario de Ensenada". Existe una copia incompleta en la Real Academia de la Historia, ms. 9/6.258.

(28) Cfr. R.A.H., "Censo de Aranda", ms. 9/6.150. 
Por último, el padrón de 1773 (29) recoge 11.653 vecinos, una cifra algo inferior a la de 1769 , que con toda probabilidad debería interpretarse más en función de las ocultaciones inherentes a los recuentos orientados a la leva militar, que como resultado de un cambio en la tendencia demográfica.

\subsection{Avanzando en la interpretaciôn}

La aplicación de tasas variables a las series biológicas permite perfilar también una clara tendencia ascendente, si bien en ambos casos nos quedará la duda de si los valores absolutos se sitúan o no en el nivel real. Lo que parece probable es que en 1715 la población de Cádiz hubiese consolidado ya los 40.000 habitantes, aproximadamente los mismos que tenía en el primer lustro del siglo (30), antes de que tuviera lugar la depresión de 1709. Será a partir de 1725 cuando se superen las cotas máximas del reinado de Carlos II, con más de 43.000 habitantes (31). Es ahora cuando podemos considerar cerrado el breve ciclo depresivo con que se inicia el siglo y cuando debemos situar el comienzo de la tendencia al crecimiento sostenido, dominante - como ya sucediera en la anterior centuria- en el XVIII gaditano.

TABLA 2

POBLACION DE CADIZ ENTRE 1717 y 1773

Aplicando el coeficiente

a los padrones y censos

20.215

35.500

49.210

64.838

58.265

\section{Años}

1717

1723

1752

1769

1773
Aplicando tasas variables a las series parroquiales
41.500
43.000
55.000
65.000
67.500

(29) A.M.C., Sección Padrones. Leg. 1.006-1.007. "Padrón de 1773". Es rechazable la reconversión en 46.612 individuos, realizada en el mismo documento aplicando el coeficiente 4 .

(30) Según nuestros cálculos, resultan demasiado cortos los 30.000 que se proponen en DOMINGUEZ ORTIZ, A.: Sociedad y Estado en el siglo XVIII español, Madrid, 1976 , p. 228.

(31) En 40.000 habitantes se estima la población de cádiz a la muerte de Carlos II, si bien en los últimos años de su reinado pudieron rebasarse los 42.000 , Cfr. KAMEN, H,: La España de Carlos II. Barcelona, 1981, p. 232. La misma cifra; tomada de la Theórica..., de Ustáriz, se aporta en ANTON SOLE, P.: "Datos básicos para la historia de la Diócesis de Cádiz en el siglo XVII". Gades, 1 (1978), p. 91. 
Los resultados obtenidos empleando el coeficiente conversor de vecinos en habitantes y aplicando tasas variables a las series biológicas manifiestan ahora una mayor similitud. Si exceptuamos la cifra del vecindario de 1717 , abiertamente deficitaria, las diferencias entre las series obtenidas por ambos procedimientos oscilan entre el 10 y el 17 por ciento, alcanzándose la plena identidad en el cálculo correspondiente a 1769. No cabe duda de que ello se debe al progresivo ajuste de las estimaciones censales a medida que nos adentramos en el siglo XVIII, cada vez más precisas y rigurosas en lo que se refiere al procedimiento de elaboración. Del 51 por ciento en que podemos situar las deficiencias del Vecindario de Campoflorido pasamos al 17 por ciento en 1723 , al 10 por ciento en 1752 y al insignificante 0 ' 2 por ciento en que estimamos el déficit en 1769.

En cuanto a la tendencia, ambas series manifiestan un ritmo de crecimiento sostenido. Habría, no obstante, que ilustrar el final del período con una aclaración, pues el padrón de 1773 recoge 11.653 vecinos, un 10 por ciento menos de los que cabría atribuir al censo de 1769 por aplicación del coeficiente 5 (32). Es impensable que en pleno auge del comercio americano (33), y en sólo cuatro años, pudiera darse una pérdida similar, evaluable, si creemos al padrón —que habla de 46.612 individuos-, en más de 18.000 personas; más aún, si tenemos en cuenta que las series biológicas no reflejan contracción alguna (34). La clave para interpretar estas discordancias está en la diversidad de criterios con que se llevan a cabo las dos estadísticas, ya que, mientras que la de 1769 incluye a toda la población urbana, la de 1773, sólo contabiliza a los nacionales establecidos no exentos del reemplazo. Y sabemos que la población flotante de Cádiz era muy elevada en estos años. Muy probablemente en la aceptación acrítica de la cifra aportada por el padrón de 1773 esté el origen de una idea tan generalizada como inconsistente, a saber: sobredimensionar los efectos del traslado del Departamento de Marina a la Isla de León

(32) Si empleásemos, como se hace en el propio padrón, el coeficiente 4, la rebaja se elevaría nada menos que a un 28 por ciento, a todas luces inverosímil.

(33) Vid. GARCIA-BAQUERO, A.: Cádiz y el Atläntico (1717-1778), I. Sevilla, 1976, pp. 540-556.

(34) En 1769 se inscriben 1.838 nacimientos y 439 matrimonios, mientras que en 1773 los nacimientos ascienden a 1.915 y los matrimonios a 457, Cfr. PONCE CORDONES, F.: "Dos siglos claves..." op. cit., p. 443. 
en 1769 , de la que el propio A. de Castro se convierte en vocero y difusor, estimando en "16.000 ó más" las pérdidas que hubo de sufrir Cádiz por este hecho (35).

En suma, parece demostrado que entre 1714 y 1773 la población gaditana crece ininterrumpidamente. La cifra de 46.612 habitantes para 1773, "completada" de acuerdo con lo expuesto (retomando el criterio contable de 1769), se elevaría hasta unas 67.500 almas, lo que nos permite evitar el absurdo de admitir que entre 1717 y 1773 la población sólo hubiera aumentado algo más de 5.000 personas, con una tasa del 0'21 por ciento, inferior incluso a la que da J. Nadal para el conjunto de España en el mismo período (0'42) (36). Por contra, sabemos que Cádiz en este siglo se convirtió en una de las más populosas ciudades españolas (37), en la cual los propios regidores consideraban una tarea "quassi impractible [...] llevar al cabo un asunto tan crítico, y tan difícil de verificar [como es un padrón]", pues en ella "concurren todas quantas circunstancias son imaginables" (38). Y, en efecto, así era; más si se trataba de confeccionar la estadística del reemplazo (39).

Aceptando las cifras que se obtienen aplicando tasas variables a las series biológicas (40), obtenemos un total de 67.500 habitantes para 1773 , lo que se ajusta bien a los 68.000 que propone Ponce para 1770-1775 (41). Una cifra, por tanto, bastante aceptable para tomarla como referencia del crecimiento demográfico gaditano en la etapa de plenitud. Entre 1717 y 1773, la población se habría incrementado en 26.000 individuos, un $62^{\prime} 65$ por ciento, con una tasa media de crecimiento anual para todo el período de 0'87 por ciento, valores todos ellos que concuerdan bien con lo que debió ser la gran expansión de la ciudad en esos años.

Pero dentro del período posterior a 1714 es posible detectar diversas fluctuaciones en el ritmo de crecimiento:

(35) CASTRO, A. de: Memoria histórica de la población y la estadística en la provincia de Cádiz, Cádiz, 1862, p. 16.

(36) Cfr. NADAL OLLER, J.: La población española... op. cit., p. 89.

(37) Cfr. DOMINGUEZ ORTIZ, A.: "Cádiz en la historia moderna de Andalucía". En: Cádiz en su bistoria. I Jormadas de Historia de Cádiz, abril 1982. Cádiz, 1983, p. 14.

(38) A.M.C. Actas Capitulares. Leg. 129. Año 1773, f. 205.

(39) Así se hace constar al final de cada cuaderno de barrio, amenazando con duras sanciones para los que falseasen los datos encuestados.

(40) Aplicamos $\mathrm{N}_{173}=28$ '5 por mil y $\mathrm{C}_{177}=6{ }^{\prime} 7$ por mil, según las tendencias acusadas en la natalidad y la nupcialidad. Ponce, por su parte, estima $\mathrm{N}_{173}=27$ por mil y $\mathrm{C}_{3}=7$ '5 por mil.

(41) Cfr. PONCE CORDONES, F.: "Dos siglos claves..." op. cit., p. 428. 
a) Entre 1714 y 1723 se produce un importante salto que eleva el volumen de población de 39.500 a 43.000 habitantes, lo que significa que se crece a un ritmo del 0'95 por ciento anual. No obstante, el principal avance se produce, como es habital en las salidas de las crisis, en los primeros años (1714-1717), donde la tasa alcanza el 1'66 por ciento, para luego decrecer hasta 0'59 por ciento entre $1717 \mathrm{y}$ 1723. En términos absolutos la recuperación supone un incremento de casi el 9 por ciento.

b) Entre 1723 y 1752 la población crece en valores brutos, pasando de 43.000 a 55.000 habitantes, con una tasa de 0'85 por cuento. Sin embargo, las series biológicas permiten detectar que el ritmo se hace cada vez menos intenso. Se trata de un crecimiento perceptible (27'91 por ciento), pero variable en su ritmo. No hay que olvidar que en esta etapa tiene lugar en Cádiz una nueva y grave epidemia de fiebre amarilla (1730), cuya mortalidad rebasó el millar de individuos, y que, contra lo que opina Ponce, debió traducirse sensiblemente en el volumen de la población (42). El efecto selectivo del vómito negro sobre los grupos de edad intermedios (fértiles) (43), explica la ralentización del crecimiento a partir de 1730 y la contracción que parece dominar el medio siglo gaditano. A ésta hay que añadir otras epidemias de menor agresividad acaecidas en 1733 y 1744 (44). El crecimiento lento, por otra parte, coincide con el ritmo que atribuye García-Baquero al comercio indiano en este período (45). En la década de 1740 el estancamiento demográfico parece incluso trocarse en abierta recesión.

c) Entre 1752 y 1773 el signo de la tendencia vuelve a ser, con mayor firmeza, positivo. Se pasa de 55.000 a 67.500 individuos. La subida absoluta es del $22 ' 73$ por ciento y se produce con un ritmo muy intenso, reflejado por una tasa de crecimiento medio anual del 0'98 por ciento. La etapa se inicia con gran vigor, relacionado, sin duda, con el excelente momento que atraviesa el comercio americano (46). El crecimiento medio hasta 1769 es alto, arañando el 1 por ciento, y a partir de entonces se desacelera ligeramente, bajando al 0'95

(42) Vid. Ibídem. p. 429.

(43) Vid. AREJULA, J.M.: Breve descripción de la Fiebre Amarilla padecida en Cádiz y pueblos comarcanos en 1800, en Medina-Sidonia en 1801, en Málaga en 1803, y en esta ültima plaza y varias otras del Reyno en 1804. Madrid, 1806.

(44) Cfr. GONZALEZ DE SAMANO, M.: Monografía bistórica... op. cit., p. 31.

(45) Cfr. GARCIA-BAQUERO GONZALEZ, A. Cádiz y el Atläntico..., I, pp. 540-549.

(46) Vid. Ibídem, pp. 550-556. 
por ciento hasta el final del período. Esta leve oscilación sitúa en su justa medida las secuelas de dos hechos a menudo considerados muy negativos para la demografía local: la epidemia de fiebre amarilla de 1764 (47) y el ya mencionado traslado del Departamento de Marina, cuyos efectos no fueron en absoluto tan devastadores como se ha pensado (48).

\section{TABLA 3}

TASAS DE CRECIMIENTO EN EL PERIODO 1693-1773 (49)

$\begin{array}{ccc}\text { Años } & \text { Población } & \text { TCA (en \%) } \\ 1693 & 42.500^{*} & \\ 1709 & 30.000 & -2^{\prime} 15 \\ 1710 & 32.500 & 8^{\prime} 33 \\ 1713 & 36.500^{*} & 3^{\prime} 94 \\ 1714 & 39.500^{*} & 8^{\prime} 22 \\ 1717 & 41.500^{*} & 1^{\prime} 66 \\ 1723 & 43.000^{*} & 0^{\prime} 59 \\ 1752 & 55.000^{*} & 0^{\prime} 85 \\ 1769 & 65.000^{*} & 0^{\prime} 99 \\ 1773 & 67.500^{*} & 0^{\prime} 95\end{array}$

\section{LA ULTIMA FASE DEL CRECIMIENTO}

A comienzos de la década de 1770 contaría Cádiz con una población de más de 65.000 habitantes, incorporándose al grupo de las ciudades españolas más pobladas. Unos años más tarde, si aceptamos la evaluación de Domínguez Ortiz, estaría situada ya en el cuarto lugar, "al par de Valencia, superada por muy poco por Barcelona y Sevilla y duplicada o poco más por Madrid" (50). El factor decisivo que explica este rápido e importante crecimiento es, sin duda, el flujo regular de

(47) El conde de Maule señala ya que esta epidemia apenas tuvo incidencia sobre la demografia local, Cfr. PASCUA SANCHEZ, $M^{2}$.J. de la: "Cádiz y su provincia..." op. cit.., p.295.

(48) Vid. crítica en PEREZ SERRANOJ.: La población de Cádiz en el tránsito del Antiguo al Nuevo Régimen (1775-1835). Cádiz, 1990, tesis doctoral inédita.

(49) Las cifras marcadas con (") globalmente homologables con las que aporta Ponce Cordones en "Dos siglos claves..." op. cit., p. 428. No obstante, a partir de 1764 los datos de nacimientos y matrimonios que este autor ofrece son deficitarios, pues no tiene en cuenta los valores de la parroquia castrense.

(50) DOMINGUEZ ORTIZ, A.: "Cádiz en la historia moderna de Andalucía." En Câdiz en su bistoria. IJornadas de Historia de Cádiz, abril 1982, Cádiz, 1983, p. 14. 
este rápido e importante crecimiento es, sin duda, el flujo regular de inmigrantes que acudirían a la ciudad atraídos por la intensa actividad de su comercio. Proliferan los testimonios directos de ello, como el del viajero J.F. Peyron referido al año 1772-1773, quien nos relata con asombro cómo "los comerciantes extranjeros de todos los países de Europa, se encontraban en multitud en Cádiz", lo que explica porque "Cádiz era el centro hacia el que todas las fortunas del reino se dirigían: el comercio hallaba alli recursos inagotables" (51). Tales eran pues las óptimas condiciones en que inicia Cádiz el último cuarto del siglo XVIII. Todavía entonces resultaban inconcebibles las imágenes dantescas del verano de 1800.

\section{1. ¿Qué dicen las fuentes?}

En el último cuarto del XVIII sólo se efectuó un recuento general de la población gaditana, el padrón de 1786, realizado en cumplimiento de la orden de 25 de julio que dio lugar al censo de Floridablanca (52). Sin embargo, por el protagonismo de Cádiz en todos los órdenes durante esta etapa - derivado de su privilegiada función en el intercambio euro-americano-, contamos con numerosas referencias relativas a su población, cuyo volumen y diversidad eran los elementos que más poderosamente llamaban la atención de los observadores. Suponemos también que al filo del inicio del periodo, en 1773, el número de pobladores estaría en torno a los 67.500 , una vez criticado el dato del padrón de quintas de ese año; asimismo, contamos con la cifra de 57.837 habitantes para finales de 1801 (53). Entre ambos, el resultado del propio recuento de $1786,71.499$ individuos, aclarando que "no se incluye la Tropa de la guarnición, ni los Cuerpos Militares que residen en esta Ciudad, como ni tampoco los Habitantes de su Bahía" (54). Hasta aquí lo que dicen las fuentes demográficas directas, muy escuetas, pero extraordinariamente bien situadas en el tiempo, pues permiten percibir los dos grandes sesgos del movimiento demográfico en estas décadas: la última fase del crecimiento, que se extiende hasta los años 90, y el brusco cambio de tendencia que acaece en el fin de siglo.

(51) Cfr. PASCUA SANCHEZ, M" J. de la: "Cádiz y su provincia..." op. cit., p. 301.

(52) A.M.C., Sección Padrones. Leg. 1.008. "Padrón de 1786". El censo de 1787 fue el primero impreso en España: Censo español executado de orden del Rey comunicada por el Excelentísimo Señor Conde de Floridablanca, primer Secretario de Estado y del Despacho, en el año de 1787. Madrid, Imprenta Real, [s.a.]. Puede consultarse en la Biblioteca Nacional, sig. R/36.402.

(53) A.M.C., Sección padrones. Leg. 1.029. "Padrón de 1801".

(54) A.M.C., Sección Padrones. Leg. 1.008. "Padrón de 1786". [Resumen], s.f. 
Por lo que se refiere a los testimonios historiográficos, sobre el comienzo del período recogemos, con inicial extrañeza, la cifra de 15.567 "habitantes" (sic) que nos proporciona A. Castro para 1773 (55). Es seguro que se trata en realidad de "vecinos", aunque el padrón de ese año contabiliza sólo 11.653 , pero, puesto que éste último es sólo un cómputo parcial, cabría no desestimar la opinión de Castro, máxime cuando ésta es perfectamente homologable con la rectificación que hemos hecho del dato de 1773. Si aplicamos la misma relación (4'3) al resultado del recuento de 1769 todo encaja mejor, ya que el incremento en estos cuatro años sería de 488 vecinos, plenamente aceptable, y no de 2.599 como habria que aceptar de mantener el coeficiente 5 para reconvertir el dato de 1769 , lo que equivaldría a atribuir a este último quinquenio una tasa de crecimiento anual del 4'67 por ciento, completamente desmesurada para Cádiz en esos años.

Probablemente la evaluación del número de vecinos de Cádiz en 1773 que transmite Adolfo de Castro haya servido de base al profesor Domínguez Ortiz para, empleando un coeficiente quizás excesivamente alto, situar en 73.000 habitantes la población urbana en 1775 (56). A nuestro juicio esta actuación se explica porque el autor sobredimensiona el ritmo y el volumen del crecimiento de Cádiz en los siglos XVII y XVIII, pues adjudica una cifra de sólo 7.000 habitantes a 1650 y, por contra, eleva a 70.000 la población un siglo después, lo que dispara la tasa de crecimiento medio anual (2'93 por ciento). Más verosímil resulta el cálculo de I, Porquicho, que fija en 21.900 los habitantes a mediados del XVII (57), y ya hemos visto que no parece probable que en torno a 1750 Cádiz rebasase las 55.000 personas; de este modo, la tasa se situaría en un nivel más razonable (0'93 por ciento). Así pues, resulta bastante atinado el cálculo de Ponce Cordones, que estima en 68.000 habitantes el volumen de la población hacia 1775 , aplicando tasas variables a las series biológicas (58).

Aunque para 1786 contamos con una fuente directa que nos

(55) CASTRO, A. de: Memoria bistórica... op. cit., p. 18. A continuación nos dice que "extramuros [apenas el 1 por ciento de la ciudad...] no constaba más que de [...] 332 vecinos", lo que por sí solo demuestra la incoherencia de la cifra global de 15.567 "habitantes".

(56) Cfr. DOMINGUEZ ORTIZ, A. (ed.): Historia de Andalucia, VI. Barcelona, 1981, p. 147.

(57) Cfr. PORQUICHO MOYA, I.: Cádiz. Población... op. cit., p. 199.

(58) Cfr. PONCE CORDONES, F.: "Dos siglos claves..." op. cit., p. 433. 
habla de 71.499 habitantes, es importante señalar que ya A. de Castro plantea la necesidad de aumentar esta cifra con la incorporación de los militares de mar y tierra y las tripulaciones de los buques fondeados en la Bahía (59). Aunque el historiador gaditano hizo gala de muy buen sentido, pues los militares no incluidos en el padrón de 1786 pueden en efecto estimarse con cierta aproximación tomando como referencia su número en 1801, no es éste el caso de las tripulaciones matriculadas en el puerto, por lo que no sería riguroso afirmar -como se hace- que en la cifra de 74.500 individuos están incluidos estos últimos (60). Sobre el número de vecinos es necesario también precisar que el difundido por Bernal, Collantes y García-Baquero, 16.635, no es exacto, pues ese volumen corresponde sólo a los barrios ubicados en el interior de la muralla, excluyéndose incluso de ellos a los vecinos asentados en conventos y obras pías (61). El recuento de 1786 recoge un total de 16.885 vecinos para el conjunto de la ciudad, que es el dato con el que relacionar los 71.499 inscritos en el padrón.

Para la década de 1790 contamos con diversos testimonios. Aunque dedicándole elogios tales como ser "Pueblo de mucho luxo, de mucho vecindario [...] y abundante de dinero", creemos que resulta manifiestamente corta la cifra de 70.000 personas que adjudica $\mathrm{A}$. Ponz a Cádiz para los primeros años de la década (62). R. Solís nos dice que la ciudad tenía 74.500 habitantes en 1791, no sabemos -pues no lo aclara - si como resultado del análisis de los datos que ofrece Gervasio de Artiñamo acerca de los extranjeros presentes en Cádiz en esa fecha (63) o, simplemente, por efecto de añadir al total

(59) En su Memoria bistórica... op. cit., p. 21, puede deducirse esto, pero en el texto nunca figura la cifra de 74,500 como afirma J.M. Rodríguez Gordillo en "Las crisis demográficas gaditanas de mediados del siglo XIX (Las epidemias de 1854 y 1856)". Gades, 1 (1978), p. 141.

(60) La guarnición comprendía 2.938 individuos en 1801; los "habitantes de la Bahía" se desconocen en ambos casos, Cfr. PEREZ SERRANO, J.: La población de Cádiz..., op. cit., p. 63.

(61) Cfr. BERNAL, A.M.. -COLLANTES DE TERAN, A- GARCIA-BAQUERO, A.: "Sevilla..." op. cit., p. 23.

(62) PONZ, A.! Viage de España en que se da noticia de las cosas más apreciables, y dignas de saberse que bay en ella, XVIII. Madrid, 1794, p. 13.

(63) Cfr. SOLIS LLORENTE, R.: El Cádiz de las Cortes. La vida en la ciudad en los años de 1810 a 1813. Barcelona, 1978, p. 66. Solis toma la cifra de extranjeros de ARTIÑAMO, G. de: Historia del comercio con las Indias durante el dominio de los Austrias. Barcelona, 1917. No obstante, la cifra que ofrece (8.734) debe ser cotejada con la más modesta resultante del recuento directo (unos 5.000). Cfr. A.M.C., Sección Padrones. Leg. 4.941. "Padrón de 1791". 
de 1786 la guarnición excluida en la estadística. En cualquier caso, lo que parece claro es que en estos años la ciudad, manteniendo aún la tendencia ascendente que caracteriza la centuria, no sólo no presenta síntomas de estancamiento, sino que podrá ver incrementado todavía en algunos miles el número de sus pobladores. Eso es lo que se desprende también de una estadística oficial de comienzos del XIX, que, refiriéndose al período que va de 1786 a 1800, afirma que "no es dudable que en este intermedio haya habido época en que su vecindario aumentase de 4 a 5 mil almas" (64). Si aceptamos esto, y las series confirman el progreso, habría que admitir que en el primer lustro de los 90 Cádiz debió encontrarse por encima de los 75.000 habitantes, aunque sin romper la frontera de los 80.000 (65).

Hacia 1796 comienzan a manifestarse los efectos del cambio en la coyuntura política sobre el tráfico americano $y$, por ende, sobre la demografía urbana. La Sociedad Económica Gaditana de Amigos del País evalúa la población de Cádiz en el último lustro del siglo, en plena guerra hispano-británica, en 71.499 personas, 14.299 de las cuales serían vecinos (66). No se incluye la tropa, pues el dato ha sido extrapolado de 1786, pero las cifras llaman la atención, pues aunque el total de individuos es idéntico, el número de vecinos que se atribuyen a 1799 es sensiblemente inferior; explicar esta disparidad, tal vez debida sólo a la imprecisión de esta fuente indirecta, nos obligaría a rastrear qué tipo de transformaciones habrían debido producirse en las estructuras demográficas gaditanas para que, perdiéndose más de un 15 por ciento de los vecinos, pudiera la población global conservar el nivel. En todo caso, dejando a un lado esto, el hecho de considerar aplicable en 1799 el dato de 71.499 habitantes significa dar la razón a quienes en 1801 aseguraban "que en estos últimos tiempos las calamidades del comercio han influido en [...la] desmembración [del vecindario...], por [...lo] que al manifestarse la Epidemia en esta Ciudad a mediados de agosto, apenas compondría el número de habitantes del expresado año [1786]" (67).

(64) A.M.C., Sección Padrones. Leg. 1.029. "Estado general de las Personas invadidas de la epidemia padecida en esta M.N. y L. Ciudad de Cádiz, desde los primeros días del mes de agosto, hasta el 31 de octubre de 1800", s.f.

(65) Ya en otra ocasión propusimos situar la cota máxima anual del XVIII en 79.000 habitantes. Cfr. PEREZ SERRANO, J.: La población de Cádiz... op. cit., p. 87.

(66) Cfr. A.M.C., Arch. SEGAP, carp. 5.331. Según generosa comunicación de J. $\mathrm{M}^{2}$ García León.

(67) A.M.C., Sección Padrones, Leg. 1.029. "Estado general de las personas invadidas...", s.f. 
El siglo XVIII se cierra en Cádiz de manera dramática por èfecto de la feroz epidemia de fiebre amarilla que asola la ciudad en el verano de 1800. Suponemos que, como defiende el redactor anónimo del documento antes citado, la población gaditana en los últimos años del Setecientos habría descendido hasta el nivel que tenía a mediados de la década de los 80; incluso puede que hubiese rebasado este límite en su caída, aunque manteniéndose siempre — creemos - por encima de los 70.000 habitantes. Cuando desaparece la epidemia, en noviembre, las fuentes constatan que la población ha descendido vertiginosamente: se contabilizan sólo 50.112, excluidos los militares, más de 20.000 pérdidas respecto al primer semestre del año (68). En julio de 1801 se elabora un nuevo recuento general del vecindario, que arroja un total de 53.898 individuos, a los que se añaden 3.638 militares de mar y tierra y 301 marineros, es decir, 57.837 personas, lo que parece manifestar una ligera recuperación respecto a los meses finales del año anterior (69).

No obstante, las deficiencias ostensibles de este recuento (70) aconsejaron realizar una comprobación, cuya estadística quedó definitivamente fijada en diciembre del mismo año; su resultado, aunque idéntico al de julio en cuanto al total de personas, rebaja el número de los militares de 3.638 y 2.637 y traslada la diferencia (1.001 individuos) a la población civil, que se eleva así a 54.899 almas. Este cálculo puede ser tenido como el más fiable de cuantos disponemos para valorar los efectos reales de la crisis: al concluir 1801 quedaban en Cádiz 12.974 vecinos cabezas de familia, correspondientes a un total de 57.837 habitantes, incluidos en ellos 838 eclesiásticos, 2.823 extranjeros, 2.637 militares de mar y tierra y 301 marineros (71).

(68) Cfr. A.M.C., Sección Padrones, Leg. 1.029. "Estado general de las personas invadidas...", s.f. La cifra se obtiene deduciendo de 71.499 los 7.387 fallecidos y los 14.000 individuos en que se estima la emigración ocasionada por la epidemia.

(69) Cfr. A.M.C., Sección Padrones. Leg. 1.028. "Padrones de 1801 [bis]", [Resumen], s.f.

(70) Vid. A.M.C., Sección Padrones, Leg. 1.029. "Padrón de 1801", Advertencias, f.2. La cifra de personas ocupadas era muy alta, así como el número de comerciantes y militares; la comprobación llevada a cabo en diciembre confirmó que estos grupos estaban sobredimensionados.

(71) Cfr. A.M.C., Sección Padromes, Leg. 1.029. "Padrón de 1.801", [Resultado], f. 1. A. Ramos nos habla de 57.837 individuos, pero les hace corresponder 11.567 vecinos, citando referencias indirectas posteriores (A.M.C., Act. Capt. Cab., 12 de enero de 1826), Cfr. RAMOS SANTANA, A.: "La confusa demografía gaditana del siglo XIX (1800-1875)". A.U.C., III-IV (1986-1987), p. 254. 


\subsection{Hacia el cambio de tendencia}

Hemos visto que, frente a la confusión y las contradicciones en que se encontraban envueltas las fuentes demográficas relativas a las primeras décadas del siglo, en el último cuarto del XVIII existe una gran unanimidad entre lo que recogen las estadísticas, lo que puede derivarse de la observación de las series y lo que se narra en los testimonios historiográficos conservados. Todos coinciden en extender la tendencia ascendente del crecimiento demográfico hasta mediados de la década de 1790, pasando Cádiz de unos 67.500 habitantes (1773) a 74.500 (1791); es muy probable que la ciudad alcanzase las 76.000 almas antes de 1795 , su cota máxima en el Setecientos, para luego descender, primero de forma moderada, hasta 71.500 (1799), y caer finalmente en picado en 1800 , conservando sólo 50.112 individuos (72). Terminadas las convulsiones que dominan el cambio de siglo, las 54.899 personas que poblaban la ciudad a finales de 1801, excluidas siempre en estos cálculos la tropa de la guarnición y las tripulaciones de los buques, representan, por su parte, el punto de partida estable desde el que Cádiz deberá afrontar el desarrollo demográfico en las nuevas condiciones.

Si trasladamos a una tabla los datos proporcionados por las distintas fuentes, tanto directas como indirectas, obtendremos un cuadro bastante preciso del crecimiento de la población gaditana en las décadas finales de la centuria:

\section{TABLA 4}

$\begin{array}{ccc}\text { TASAS DE CRECIMIENTO EN EL PERIODO } & \text { 1773-1801 (73) } \\ \text { Años } & \begin{array}{c}\text { Población } \\ \text { TCA (en \%) }\end{array} \\ 1773 & 67.500^{*} & \\ 1775 & 68.500 & 0^{\prime} 74 \\ 1786 & 74.500^{*} & 0^{\prime} 77 \\ 1791 & 77.500 & 0^{\prime} 79 \\ 1799 & 74.500 & -0^{\prime} 49 \\ 1800 & 53.050 & -28^{\prime} 79 \\ 1801 & 57.837^{*} & 9^{\prime} 02\end{array}$

(72) En 1791 se mantiene la cifra que aporta R. Solís, 74.500 habitantes, ya que a nuestro juicio la guarnición militar no está incluida en ese cálculo. El dato de $1800,50.112$ individuos, excluye también los 2.938 militares y marineros que se contabilizan en 1801.

(73) En todos los casos se incluye ahora la guarnición militar, pero no los tripulantes de los buques matriculados en el puerto, que no formaban parte realmente del vecindario. Las cifras marcadas con un asterisco corresponden a años en que se realizan recuentos directos. 
Podemos establecer con claridad tres momentos:

1. ${ }^{\circ}$ Entre 1773 y 1791 continúa el crecimiento secular, aunque a un ritmo moderadamente inferior al observado en el tercer cuarto de siglo (decae alrededor del 20 por ciento). Se mantiene, sin embargo, la gran regularidad en las tasas que había caracterizado la etapa anterior, oscilando ahora - con muy poca desviación- en torno al 0'77 por ciento anual. La densidad bruta del municipio, que en estos años ocuparía aproximadamente 852 Has. (74), se incrementará también de forma gradual, pasando de casi $8.000 \mathrm{hab} / \mathrm{Km}^{2}$ en 1773 a más de 9.000 en 1791.

2..$^{\circ}$ En algún momento entre 1791 y 1800 tiene lugar el cambio en el signo de la tendencia, que se hace negativo ya en 1799 , produciéndose la caída vertiginosa en 1800 , con una tasa negativa de casi el 29 por ciento en este año. La densidad bruta, que en 1791 alcanzaba los $9.096 \mathrm{hab} . / \mathrm{Km}^{2}$ y que muy probablemente todavía se incrementase algo en ese lustro, manifestará ya síntomas de retroceso en 1799 , descendiendo hasta $8.750 \mathrm{hab} . / \mathrm{Km}^{2}$; esta tendencia regresiva tocará fondo en 1800 , con sólo $6.227 \mathrm{hab} . / \mathrm{Km}^{2}$ (la densidad se reduce en un $31^{\prime} 5$ por ciento respecto al momento de mayor concentración).

3..$^{\circ}$ Finalmente, en 1801 se constata una moderada recuperación de los niveles poblacionales, creciéndose en un sólo año a un ritmo superior al 9 por ciento, aunque será necesario esperar unos años para verificar la solidez de esta nueva tendencia. De igual modo, la densidad bruta del municipio se incrementa perceptiblemente respecto a 1800; ahora se alcanzan los $6.788 \mathrm{hab} . / \mathrm{Km}^{2}$, una concentración todavía por debajo de las que habían caracterizado la fase final del crecimiento, pero indicativa ya de que se ha frenado el creciente deterioro de la masa poblacional iniciado en torno a 1795.

Por lo que respecta a las densidades urbanas del casco, las más significativas, pues en este reducido espacio de sólo 107 Has. (75) se albergaba de manera estable más del 98 por ciento de la población gaditana en este período, habría que repetir lógicamente lo dicho

(74) Esta superficie, unos $8^{\prime} 5 \mathrm{Km}^{2}$, englobaría todo el término municipal, es decir: el recinto amurallado, los extramuros y el conjunto de terrenos no urbanizados que iban desde la Puerta de Tierra hasta el rio Arillo, incluidas las salinas y los suelos arenosos.

(75) La concentración urbana del casco se calcula a lo largo de todo el período sobre la superficie total del recinto amurallado, $107 \mathrm{Has}$, puesto que ya desde mediados del XVIII este espacio se había convertido en suelo ocupado o de inmediata urbanización. 
acerca de la tendencia. Sin embargo, el interés de estas cifras es otro: poner de relieve la extraordinaria - casi inaudita- concentración humana que se dio en el casco antiguo de Cádiz a finales del siglo XVIII. Baste sólo con decir que en el momento de mayor "despoblación", en 1800, la densidad en los intramuros era nada menos que de $51.950 \mathrm{hab} . / \mathrm{Km}^{2}$, lo que, siendo sin lugar a dudas una concentración espectacular, deberá valorarse teniendo en cuenta que la ciudad había llegado a rebasar los $71.000 \mathrm{hab} . / \mathrm{Km}^{2}$ en los años de esplendor. Aunque lamentablemente no podemos entrar aquí, por razones de espacio, en el análisis sistemático de esta variable por medio de los índices de urbanización (76), podemos considerar consumado el objetivo inicial de formar un primer bosquejo del movimiento demográfico. Veamos, no obstante, por último, una tabla con los valores relativos a la densidad bruta municipal y la concentración urbana (incluida siempre la guarnición militar):

TABLA 5

DENSIDADES BRUTAS Y URBANAS EN EL PERIODO 1773-1801

\begin{tabular}{|c|c|c|c|c|}
\hline Años & $\begin{array}{c}\text { Población } \\
\text { total }\end{array}$ & 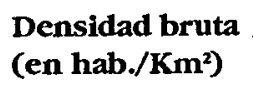 & $\begin{array}{l}\text { Población } \\
\text { del casco }\end{array}$ & $\begin{array}{l}\text { Densidad urbana } \\
\text { (en hab./Km²) }\end{array}$ \\
\hline 1773 & 67.500 & 7.923 & 66.400 & 62.056 \\
\hline 1775 & 68.500 & 8.040 & 67.400 & 62.991 \\
\hline 1786 & 74.500 & 8.744 & 73.413 & 68.610 \\
\hline 1791 & 77.500 & 9.096 & 76.400 & 71.402 \\
\hline 1799 & 74.500 & 8.744 & 73.413 & 68.610 \\
\hline 1800 & 53.050 & 6.227 & 51.950 & 48.551 \\
\hline 1801 & 57.837 & 6.788 & 56.726 & 53.015 \\
\hline
\end{tabular}

\section{CONCLUYENDO}

$\mathrm{Si}$, como hemos visto, en el extraordinario progreso experimentado por Cádiz durante la centuria dieciochesca habían jugado a favor de la ciudad la fortaleza y estabilidad política del Imperio español, en el nuevo siglo los avatares y desaciertos de la política borbónica, tanto en el ámbito interior como en la escena internacional, habrían de tener un efecto fuertemente negativo sobre la demografía local. A par-

(76) Para iniciarse en el manejo de estos índices fundamentados en el análisis matricial, a los que en otras ocasiones nos hemos referido y para obtener a la vez una buena visión de conjunto del proceso de urbanización europeo, Vid. VRIES, J. de: La urbanización de Europa, 1500-1800. Barcelona, 1987. 
tir de 1801 se inicia en Cádiz una nueva fase, definida por la incàpacidad manifiesta de su población para recuperar de manera estable los niveles demográficos alcanzados en la etapa anterior. A ello coadyuvaron diversos factores de índole política y económica, y también determinantes estrictamente demográficos, como el hecho de que la población urbana hubiera de sufrir el persistente embite de la temible fiebre amarilla, que asoló la ciudad en cuatro ocasiones durante las primeras dos décadas del siglo. No carecen de razón quienes, como J. Nadal, consideran que Cádiz "quedaría herida de muerte a consecuencia de ello" (82), si bien no debemos olvidar que el principal factor del estancamiento fue sin duda la falta de vitalidad intrínseca de la propia población y, más concretamente, el raquitismo de sus mecanismos reproductores, perceptible con claridad incluso en la fase álgida del crecimiento (83). 


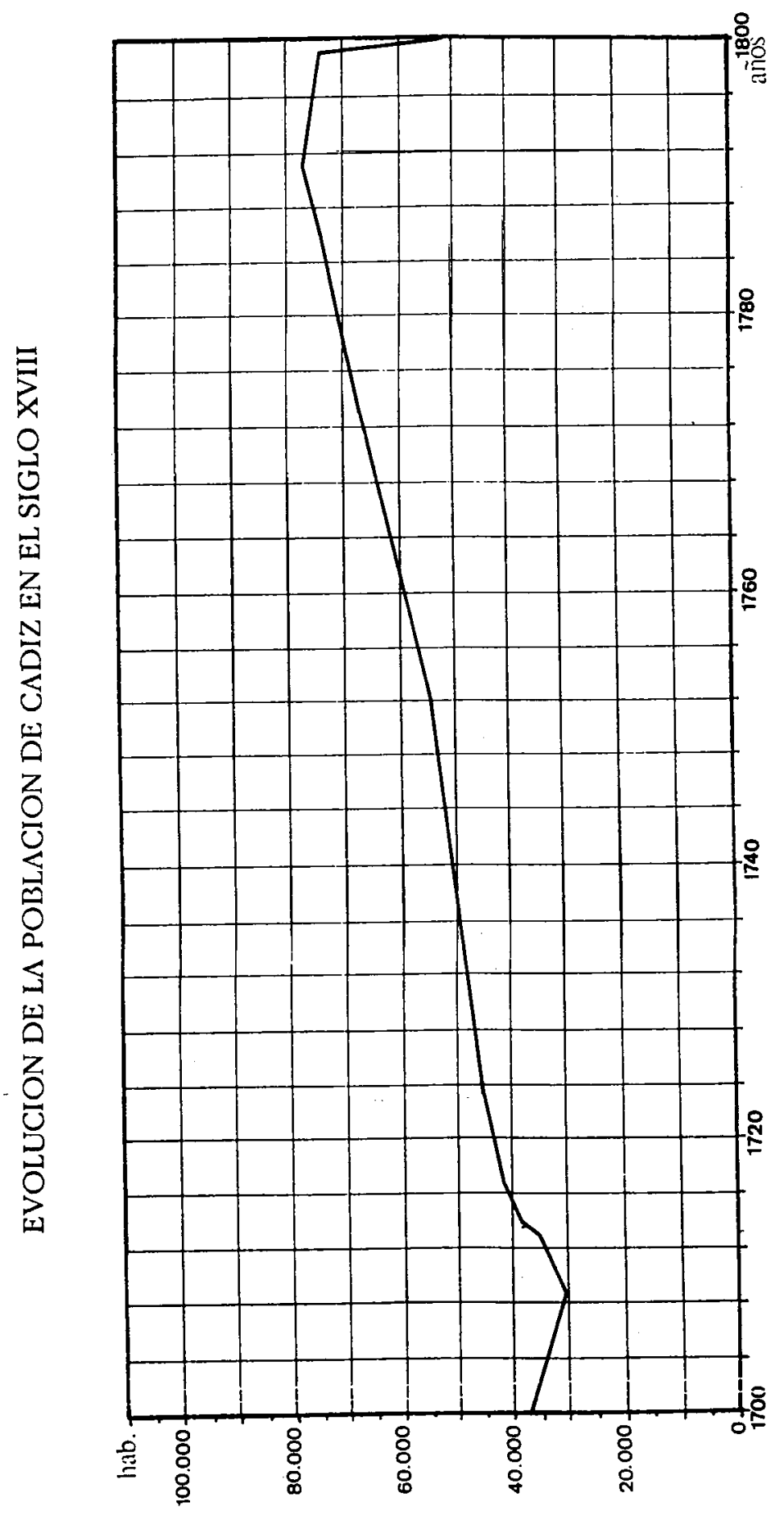

